\title{
Electrodiagnostic study in delayed facial palsy after closed head injury
}

\author{
K. PUVANENDRAN, M. VITHARANA, AND P. K. WONG \\ From the University Department of Medicine, and the Department of Otorhinolaryngology, \\ Singapore General Hospital, Singapore
}

SUMMARY The time course of electrical reactions in this condition varied much from that after section of the facial nerve or after Bell's palsy. It was at least a week before denervation could be suspected in the latter conditions by electrodiagnostic methods. In post-traumatic delayed facial palsy we have demonstrated electrical reactions showing denervation at the time of palsy or even a few days before, and this is probably related to its pathogenesis. Denervation occurred in $82 \%$ of cases which is about double the incidence in Bell's palsy.

The purpose of this study was to examine the time course of electrical reaction of the facial nerve after closed head injuries, especially the changes after delayed facial palsy, and to detect denervation early in order to help in management, particularly when surgical treatment was contemplated. The cases investigated are described by Puvanendran et al. (1977). It used to be a minimum of two weeks before electrical reactions were known to occur after nerve section; this was before Landau (1953) demonstrated, as early as four to six days after nerve section, electrical inexcitability of the distal segment after complete nerve section.

The most accessible point at which the facial nerve can be studied is at the angle of the jaw where it emerges from the stylomastoid foramen. The lesion responsible for delayed facial palsy is in the facial canal, proximal to the site where we study it. The findings in the nerve and facial muscle distal to the lesion give indirect information on the portion of nerve involved in delayed facial palsy.

If the injury causes only a conduction block of the nerve in the facial canal, stimulation of the nerve distal to this site produces contraction of the facial muscles when the patient cannot contract that muscle voluntarily. The conduction block is due to demyelination of the affected part of the nerve.

If the nerve injury in the facial canal causes degeneration of axons, nerve stimulation distal to it can

Address for reprint requests: Dr K. Puvanendran, University Department of Medicine (I), Singapore General Hospital, Singapore 3, Republic of Singapore.

Accepted 4 November 1976 elicit a response, but with increased conduction time if the degeneration involves only the fast conducting large axons. If all the axons degenerate there will be no response on nerve stimulation.

\section{Patients and methods}

Thirty-nine cases of ear bleeding after closed head injury were studied using electrodiagnostic methods. Sixteen of these cases developed 19 facial palsies some days after the head injury.

The facial nerve was stimulated at the stylomastoid foramen over the angle of the jaw. Short-duration $(0.2 \mathrm{~ms})$ square wave electric pulses were delivered by a bipolar electrode, the active electrode being held firmly in the retromandibular fossa below the external auditory meatus. The recording electrode was a concentric needle electrode placed in the orbicularis oris muscle. The oscilloscope was triggered by the stimulus, and the display on the oscilloscope was used to study latency, action potential amplitude, and the electromyogram. The following factors were studied: facial nerve latency (conduction time), action potential amplitude, threshold of excitation, the presence of fibrillations, the shape and duration of motor unit action potentials, with special attention to polyphasic potentials, and the interference patterns. All these were compared on the normal and paralysed sides. Supramaximal stimuli were used to study conduction time. In studying the threshold of excitation, the difference in nerve excitability ketween the normal and injured side was measured. This is the difference in stimulus threshold (in $\mathrm{mA}$ ) between the two sides required to evoke a muscular action potential. Nerve 
excitability difference was considered abnormal if greater than $3.5 \mathrm{~mA}$ (Laumans, 1965).

Normal values for conduction time were studied in 71 normal facial nerves which gave a mean of $3.03 \mathrm{~ms}$, SD 0.25 (mean $+3 \mathrm{SD}=3.78 \mathrm{~ms}$ ). We have taken $4.0 \mathrm{~ms}$ as the upper limit of normal for facial nerve conduction time (latency).

It was possible to divide the lesions of these patients into three groups-(1) simple conduction block, (2) partial denervation, and (3) complete denervationdepending on the electrical reactions, the presence of fibrillation (indicating denervation), reinnervation changes such as broad polyphasic potentials, the effects of abnormal reinnervation such as blink bursts, and the clinical outcome of the palsy.

\section{Results}

\section{COMPLETE DENERVATION}

Figure 1 shows the electrical changes in a characteristic case of complete denervation. The conduction time, as indicated by the facial latency, increased steadily and steeply from the day of the facial palsy to the fourth day after the palsy, and on the fifth day the nerve excitability completely disappeared. The difference in excitability between the normal and affected sides increased simultaneously with conduction velocity changes, and ran parallel with it. The amplitude of the evoked muscle action potential rapidly declined from the same time as these changes, and disappeared altogether on the fifth day when the nerve was not excitable Figures 2 and 3 show latency changes of three such cases of complete denervation



Fig. 1 Case 19-right facial palsy with complete denervation, showing latency, nerve excitability difference, and amplitude of the right facial nerve, changing with time.

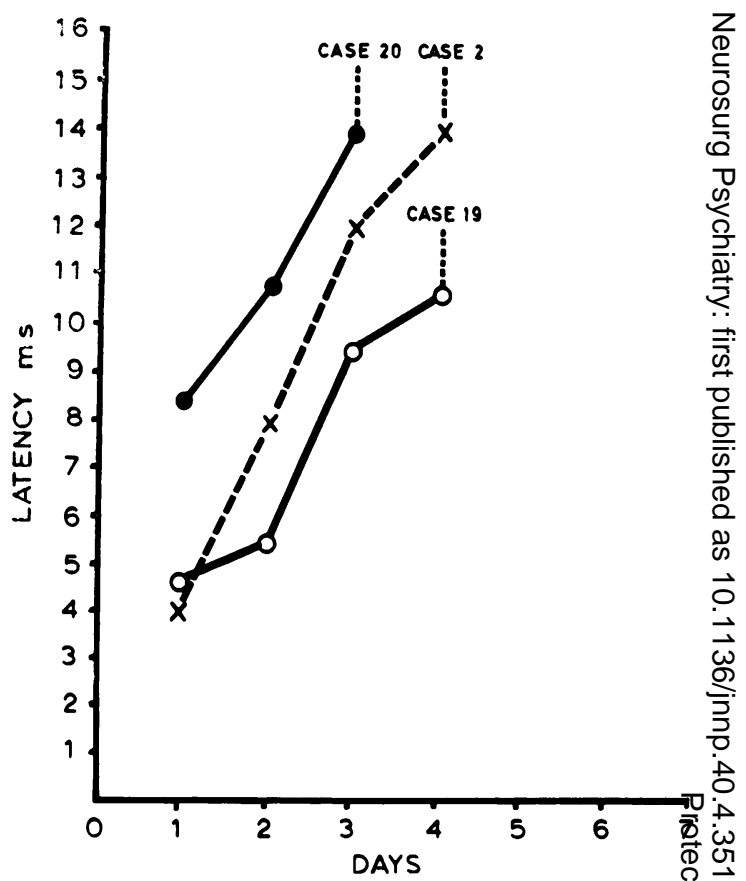

Fig. 2 Three cases of complete denervation showing their latency changing with time.

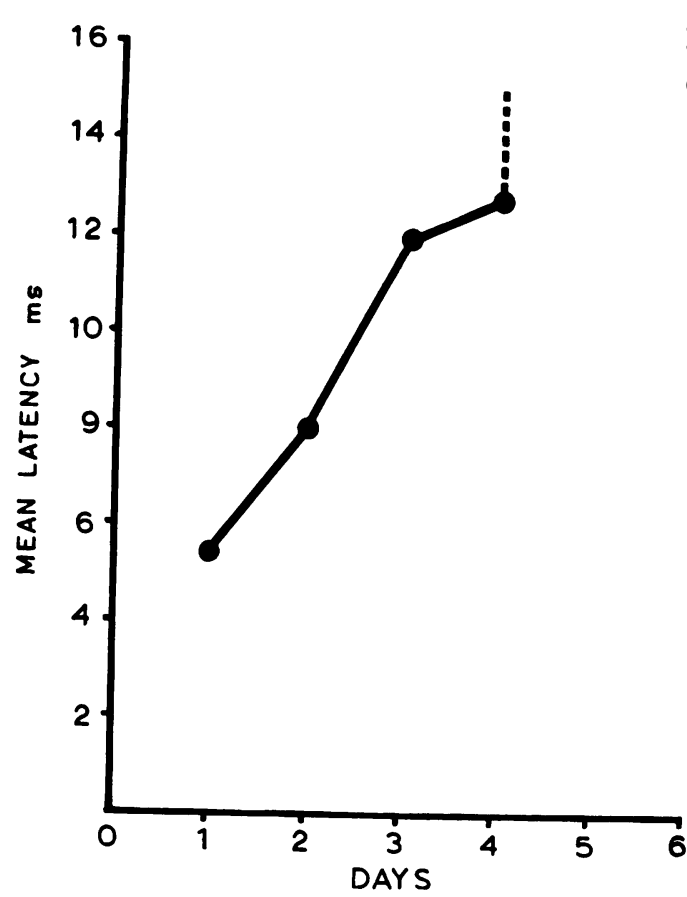

Fig. 3 Mean conduction latency in complete denervation changing with time. 
and their mean latency changes respectively. In all cases the latency increased and excitability was lost by the fifth day.

It is noted that latency changes, nerve excitability difference, and change of muscle action potential amplitude occurred almost simultaneously from the day of the facial nerve palsy. In only one case of complete denervation, when the latency increased before nerve excitability disappeared, was there no difference in nerve excitability, or changes of amplitude of evoked action potentials.

\section{CONDUCTION BLOCK}

There were no changes in amplitude of action potential or of nerve excitability differences. Figure 4 shows the latency changes with time in a typical case of conduction block (case 17). At every examination the latency was less than 4 ms (normal value). Figure 4 also shows another case of conduction block (case 15) where the latency exceeded $4 \mathrm{~ms}$ and returned to values below $4 \mathrm{~ms}$ after the 15 th day of facial palsy. In all such cases, clinical recovery was full and rapid, there was no fibrillation activity to indicate denervation and no evidence of reinnervation, such as abnormal innervation clinically or blink burst recorded by EMG, even after many months. There were two of these cases where latency exceeded $4 \mathrm{~ms}$ and then returned to normal.

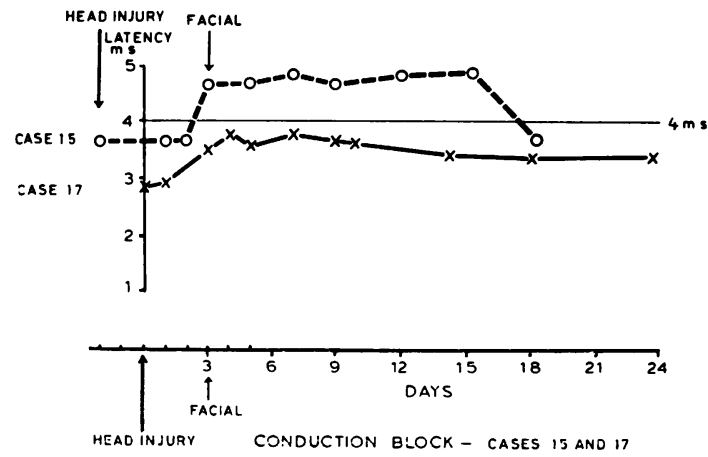

Fig. 4 Cases 15 and 17 showing conduction block and latency changes.

SUBCLINICAL PALSY (We have used this term when electrical studies indicate a nerve lesion which is not evident on clinical examination.)

In the two cases of subclinical palsies, the latency increased by about the 14th day after the head injury, and returned to below $4 \mathrm{~ms}$ by the 37th and 60th day respectively. These changes occurred simultaneously with a falling amplitude of the evoked muscular action potential and with nerve excitability changes.

\section{PARTIAL DENERVATION}

In these patients a response to nerve stimulation was always seen, and in all such patients the facial latency had exceeded $4 \mathrm{~ms}$ at some time after the facial palsy. In the typical case illustrated in Fig. 5 it will be noted that the latency had been normal after the head injury until, on the third day before facial palsy, the latency started rising above the normal $4 \mathrm{~ms}$. Figure 5 also compares the latency changes and the clinical recovery with time. About a quarter of the clinical recovery had occurred by the time the latency was reduced to normal.

Figure 6, the mean value of latency changing with time, shows that the latency is increased from the time of onset of facial palsy; it has already been pointed out that latency can increase even before the onset of the delayed facial palsy. In most cases latency came down to normal by the sixth week after the palsy. Nerve excitability difference and amplitude changes did not show any particular pattern of changes in partial denervation.

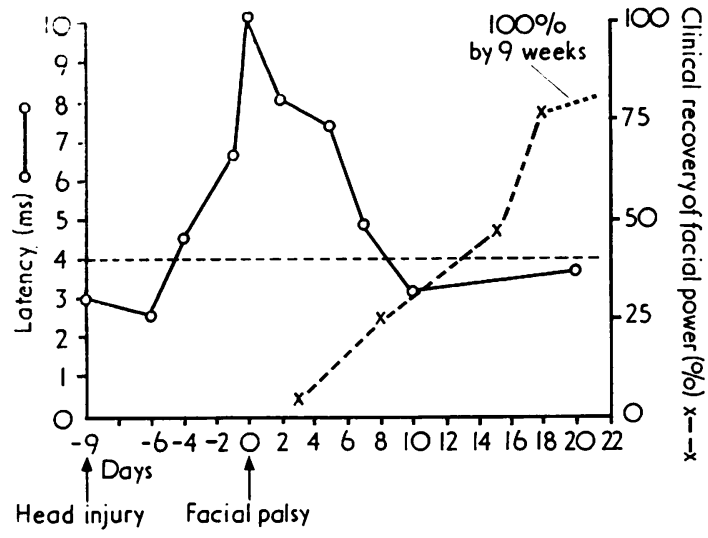

Fig. 5 Case 20 showing partial denervation. Latency changes before and after facial palsy, and time course of clinical recovery are also shown.

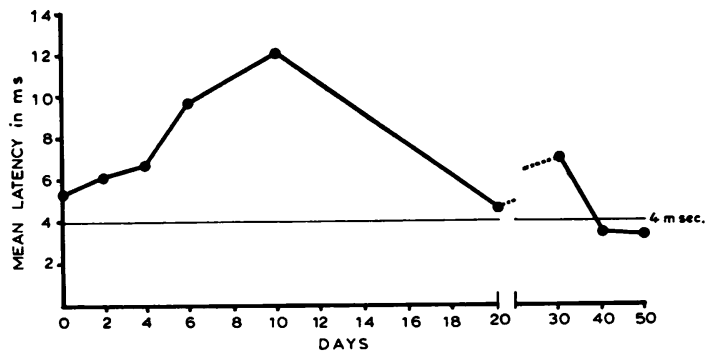

Fig. 6 Mean latency changes with time are shown in partial denervation. 


\section{REINNERVATION}

Figure 7 shows the electrical reaction during reinnervation in a typical case of delayed facial palsy which had shown complete denervation.

Conduction velocity is slowed for many days during reinnervation. Nerve excitability differences were abnormal for about $\mathbf{4 0}$ days after excitability had returned. Amplitude of the evoked response remained low even beyond 40 days. In the patient who was worst affected clinically with a complete denervation, latency returned to normal by the eighth month, but clinical recovery of power was less than $25 \%$ and by then there was evidence of abnormal reinnervation such as 'crocodile tears' and blink potentials on EMG.

\section{ELECTROMYOGRAPHY}

Fibrillation was noted in all cases of denervation except one. In this case, there was other evidence to suggest previous denervation, such as the prolonged

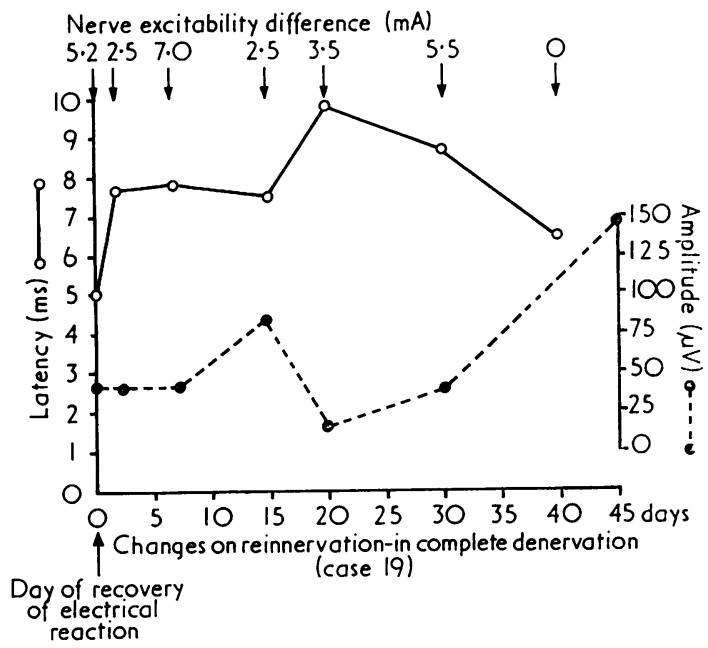

Fig. 7 Case 19 showed complete denervation. Changes during reinnervation are shown. Latency, nerve excitability difference, and amplitude are plotted. polyphasic muscle action potentials of reinnervation and other aberrant reinnervation phenomena like blink potentials. The earliest time we saw fibrillation was on the ninth day, and in some it was not until about the 75th day.

Many cases showed the prolonged polyphasic potentials of reinnervation (Fig. 8), some being about $10 \mathrm{~ms}$, and appearing long after the fibrillation potentials. In cases of complete denervation, the prolonged polyphasic potentials were the earliest signs of reinnervation, being observed a few days after appearance of nerve excitability. In cases of partial denervation they were also the earliest sign of reinnervation and appeared long before the conduction velocity returned to normal.

Spontaneous twitch of the facial muscle and 'crocodile tears' could appear before facial movements were seen. What appeared to be a spontaneous mouth twitch was in reality a twitch of the mouth synchronous with eye blinking, and this was well demonstrated on the EMG record. This phenomenon has been termed 'blink-bursts' (Taverner, 1955), and represents aberrant reinnervation (Fig. 9).

It was often found that the interference pattern with the needle in the orbicularis oris was very sparse on contraction of that muscle but became normal or voluntary contraction of another muscle such as the 을 orbicularis oculi (Fig. 10).

The blink bursts could involve different motor units from those under voluntary contraction as interpreted from the shapes of the potentials when a blink was superimposed on an interference pattern.

Sometimes when a voluntary movement was not detected visually, an almost normal interference pattern could be seen on EMG (Fig. 11).

When nerve stimulation failed to produce a facial response which could be seen, a needle in that muscle showed an action potential (Fig. 12). Thus, but for the EMG, a gloomy prognosis would be given. When there was no evoked response on stimulation of the nerve a response could sometimes be detected on voluntary movement, with a needle electrode.

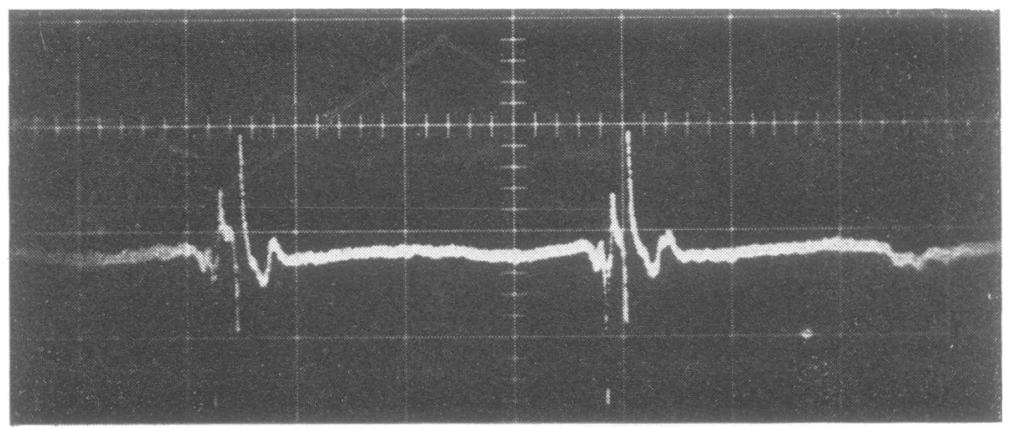

Fig. 8 Long duration polyphasic motor unit potentials of reinnervation (10 ms/division and $100 \mu$ V/division). 




Fig. 9 Blink bursts. EMG from orbicularis oris. Bursts of potential are seen on eye blinking due to aberrant reinnervation. (10 ms/division and $100 \mu \mathrm{VI}$ division).

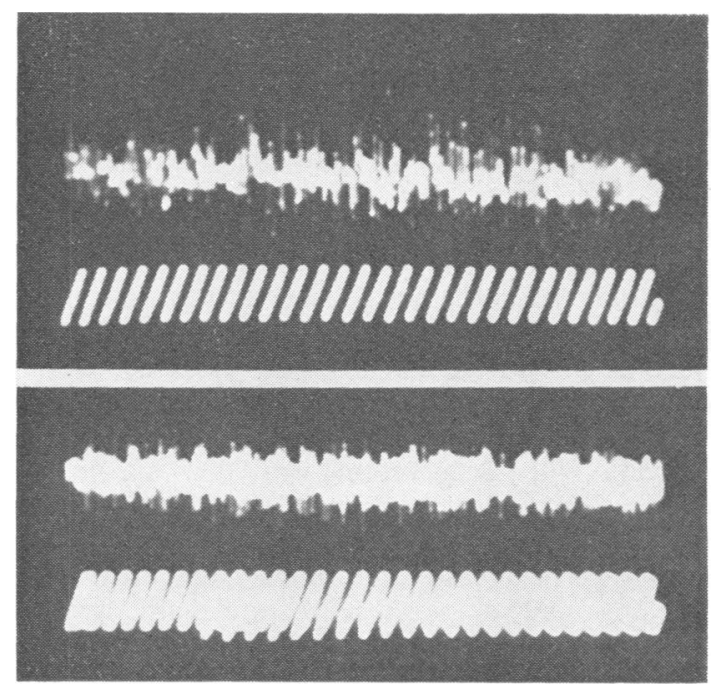

Fig. 10 Needle in orbicularis oris-good interference pattern on contracting orbicularis oculi (top), and sparse interference pattern on contracting orbicularis oris (bottom) (10 ms/division and $200 \mu$ V/division).

\section{Discussion}

The electrical reactions of the facial nerve enable one to classify a delayed traumatic facial palsy as either a conduction block, or partial or complete denervation, and give an indication of the possible outcome of the facial palsy. Cases of denervation always show incomplete recovery. If decompression of the facial nerve is contemplated it should be done early, before degeneration has advanced in order to obtain a satisfactory improvement. It is, therefore, important to be able to detect denervation at an early stage.

Complete denervation should be suspected if the

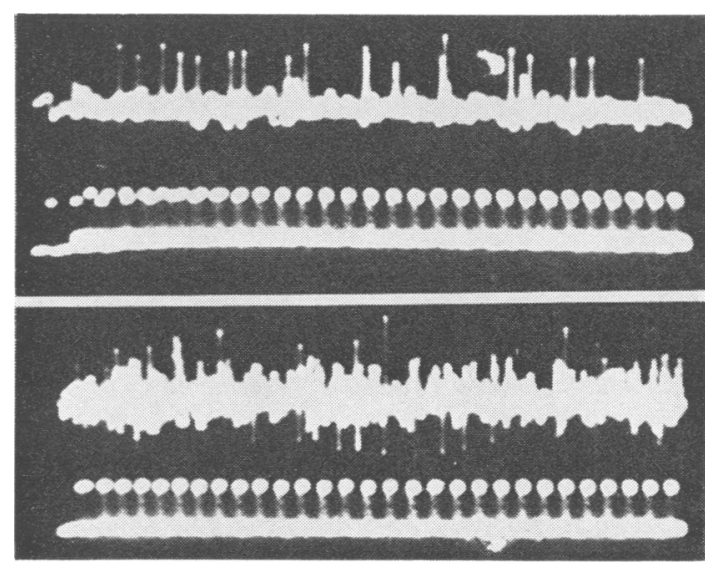

Fig. 11 Complete paralysis of right face but an EMG interference pattern could be seen in orbicularis oris (top), and normal interference pattern in normal left side (bottom), (10 ms/division and $200 \mu$ V/division).

motor latency, nerve excitability difference, and changes of action potential amplitude show a steep change, and these can be noted as early as the day after the clinical facial palsy. When no response to stimulation is obtained, complete denervation is obvious.

In the presence of conduction block and partial denervation there is always a response to electrical stimulation. Motor latency is invariably increased in partial denervation from the time the facial palsy is noted. Conduction block is usually accompanied by a normal latency but this is not always so.

The cases of subclinical palsy show a temporary increase in motor latency with simultaneous alteration of nerve excitability difference and evoked 

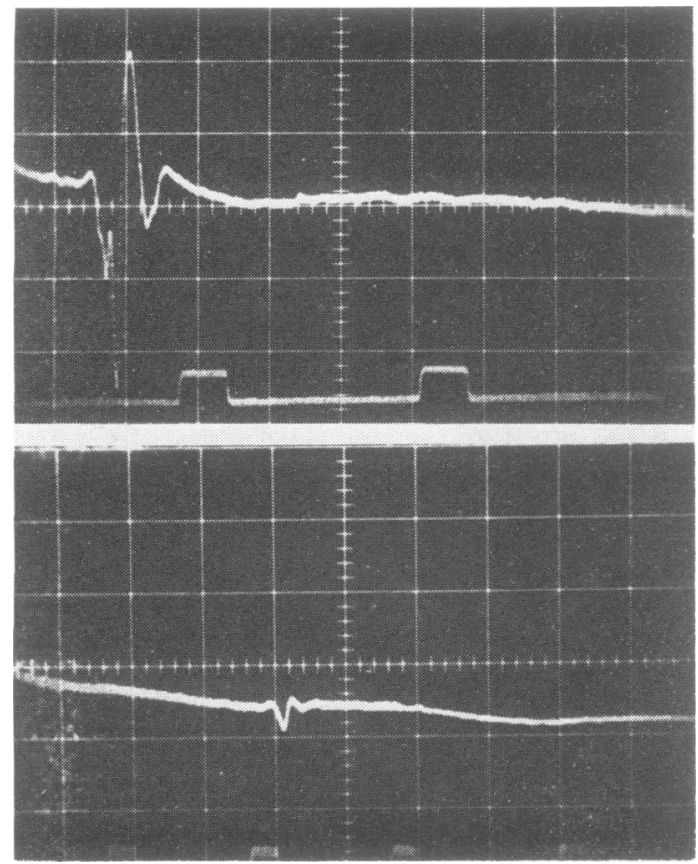

Fig. 12 Normal latency (3.6 ms) and amplitude $(1.1 \mathrm{mV})$ of evoked muscle action potential on stimulating right facial nerve (top); On stimulating facial nerve on paralysed left face no response is observed visually. A small amplitude $(75 \mu V)$ response is seen delayed by $16.4 \mathrm{~ms}$ (bottom).

potential amplitude changes (unlike clinical palsies with conduction block).

In our patients, and with our criteria, the incidence of conduction block was $17.6 \%$ and denervation occurred in $82.4 \%$.

In estimating nerve excitability, one compares the threshold of excitability on the affected side with the normal side. Evoked action-potential amplitudes are also compared on the two sides. These observations could be erroneous because we have noted subclinical palsies with altered electrical reactions occurring on the side opposite to that with a facial weakness.

In estimating the threshold of excitability, if one looks for a visible response a poor prognosis may be given for, as pointed out above, some patients who had no such response showed action potentials when a needle was used to record from the muscle.

We believe that conduction velocity is always normal in patients without denervation. In some of our patients without denervation there was definite but not severe slowing in conduction velocity. This was better exemplified by those cases where the facial latency increased a few days after the head injury, even though there was no detectable facial weakness. It is now known that compression of a nerve can produce slowing of conduction, not necessarily only over the affected part but also at a more distal point, as has been shown in compression neuropathies of the median nerve in the carpal tunnel or of the ulnar nerve at the elbow (Simpson, 1956; Gilliatt and Thomas, 1960). This would also explain why, in our patients with complete denervation, the latency increased steadily from the day the facial palsy was seen until all electrical response disappeared completely. In our patients we saw complete denervation occurring in nerves that had already for some time shown pathological electrical reactions.

Gilliatt and Taylor (1959) studying electrical reactions after facial nerve section, and Taverner (1965) studying complete denervation after Bell's palsy, stressed the point that conduction velocity did not change for three or four days and then excitability was completely lost in 24-48 hours. Laumans (1965) noted complete loss of excitability in Bell's palsy only from a minimum of 11 days after paralysis.

Gradual compression from blood in the facial canal could have caused initial segmental demyelina-o tion of the large fast-conducting nerve fibreso producing a distal slowing of conduction and at the same time causing complete denervation by secondar $\vec{D}$ ischaemia. This could also explain the shortesinterval in our patients before nerve conduction disappeared completely.

Another interesting phenomenon noted in ouk patients with denervation was that slowing of conduction in the facial nerve was detected about three days before the facial palsy was seen clinically. This could be a reason for following up patients with bleeding and a petrous fracture with frequent electrical testing if denervation is to be detected early. This reaction is also unlike Bell's palsy with partial denervation where slowing of conduction is not seen until the second week after facial palsy (Taverner, 1965). Such differences in electrical reaction between Bell's palsy and delayed traumatic facial palsy, in addition to their difference in prognosis, indicate a difference in pathogenesis in the two conditions.

The EMG was useful in showing denervation (by fibrillation) reinnervation (by presence of prolonged polyphasic potentials), and abnormal reinnervation (by blink bursts and other interference patterns). The earliest time that fibrillations were seen was the ninth day but in others it was as late as the 75th day, so its use in the early detection of denervation is limited. The problem of studying fibrillations in facial muscles is that of the involuntary movements occurring in the face, and of the short duration action potentials of the normal motor units there 
which are very like fibrillation. Also, the endplate zone occupies a greater area in the facial muscle than in other muscles, and endplate potentials are often mistaken for fibrillation. For this reason, apparent fibrillation potentials with an initial negative component should be excluded. Another difficulty with the examination of the facial muscles is that the motor latency is often found to vary with the position of the needle in that muscle. In this study, latency was taken as the shortest time recorded.

\section{References}

Gilliatt, R. W. and Thomas, P. K. (1960). Changes in nerve conduction with ulnar lesion at the elbow. Journal of Neurology, Neurosurgery, and Psychiatry, 23, 312-320.
Gilliatt, R. W. and Taylor, J. C. (1959). Electrical changes following section of facial nerve. Proceedings of the Royal Society of Medicine, London, 52, 1080-1083.

Landau, W. M. (1953). Duration of neuromuscular function after nerve section in man. Journal of Neurosurgery, 10, 64-68.

Laumans, E. P. J. (1965). Nerve excitability tests in facial paralysis. Archives of Otolaryngology, 81, 478-485.

Puvanendran, K., Vitharana, M., and Wong, P. K. (1977). Delayed facial palsy after head injury. Journal of Neurology, Neurosurgery, and Psychiatry, 40, 342-350.

Simpson, J. A. (1956). Electrical signs in the diagnosis of carpal tunnel and related syndromes. Journal of Neurology, Neurosurgery, and Psychiatry, 19, 275-280.

Taverner, D. (1955). Bell's palsy. Brain, 78, 209-228.

Taverner, D. (1965). Electrodiagnosis in facial palsy. Archives of Otolaryngology, 81, 470-477. 